\title{
Recollection
}

\section{Yinglai Wang: an admirable biochemist for his foresightedness and selflessness}

\author{
Baoyuan Zhang ${ }^{1}$, Pangao Xue ${ }^{2}$, Enduo Wang ${ }^{3}$ \\ ${ }^{1}$ Beijing Institutes of Life Science, Chinese Academy of Sciences, Beijing 100101, China \\ 2 Bureau of Life Science and Biotechnology, Chinese Academy of Sciences, Beijing 100864, China \\ ${ }^{3}$ Shanghai Institutes for Biological Sciences, Chinese Academy of Sciences, Shanghai 200031, China
}

On July 5, 2001, Nature published an article: "Wang Ying-lai (1907-2001)" in honor of Dr. Yinglai Wang and his contributions to the advancement of total chemical synthesis of biological molecules. It is rather rare for Nature to describe a biochemist in this manner. However, Yinglai Wang is an admired and revered scientist, and as such, deserves such an honor! His scientific achievements and exemplary morality should be acknowledged by the scientific community and must be emulated by people from all walks of life.

Yinglai Wang, Ph.D. of Cambridge University, UK, and Academician of the Chinese Academy of Sciences (CAS), was one of the founders of biochemical research in China. His most significant scientific contribution was the relevant leadership he provided in the first total chemical synthesis of crystalline bovine insulin and yeast alanine tRNA. His academic achievements were inseparable from his unique organizing ability and self-giving dedication.

In the early days of the People's Republic of China (1949-1957), biochemical research was unorganized. The country lacked qualified scientists and technicians. Realizing the need to change this situation, Yinglai Wang sent letters to some well-known Chinese scientists working overseas in a systematic way, and summoned them to return to China. His reasons were based on the disciplinary developmental trend of biochemistry. Consequently, from 1951 to 1957 , he succeeded in recruiting prominent scientists from abroad including Chenglu Zou, Tianqin Cao, Yourui Zhang, Debao Wang, Jingyi Niu, and Guangyu Zhou, among others, and established the Shanghai Institute of Biochemistry at CAS. In order to support and highlight the scientific research careers of his best research assistants, namely, Qinrong Wu and Jiamu Peng, he introduced them to Chenglu Zou and Tianqin Cao, respectively. The esteemed biochemist's selfless spirit and untiring encouragement transformed the Shanghai Institute of Biochemistry into a strong and qualified institution

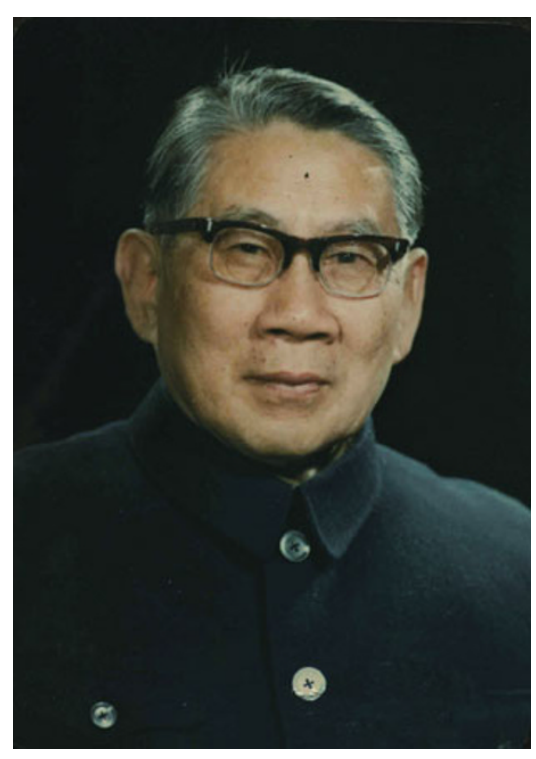

Dr. Yinglai Wang (1907-2001)

capable of completing national key scientific research projects. Under his guidance, the institute enjoyed a democratic and harmonious academic environment.

To solve the problem of amino acid scarcity for insulin synthesis, Yinglai Wang founded the Dongfeng Biochemical Reagent Factory in 1958. Under his leadership, the factory produced amino acids and various biochemical reagents to meet the whole country's need. Forty years before the advocacy for knowledge innovation and the acceleration of industrialization of scientific findings was initiated, he had already championed the model of and worked toward the integration of enterprises, universities, and institutes. Truly, Yinglai Wang was a visionary!

The Great Leap Forward Movement (1958-1960) started in 
China at a time when Yinglai Wang and other scientists had just succeeded in insulin synthesis. This inspired hundreds of scientific researchers to participate in protein synthesis research. They refocused their priorities, stayed in laboratories, and worked under stringent conditions as ordinary workers. However, these failed to take effect on the basic law of scientific research; while in order to sustain people's enthusiasm, no one opposed the launching of such mass movement. Driven by a strong sense of responsibility of a scientist, Yinglai Wang took the opportunity of attending international conferences to present his opposition to the CAS administration. His proposal was favored by CAS leaders, and made numerous people realize that a mass movement is inappropriate for scientific research. He reiterated that a capable contingent of scientists and meticulous and thorough work are vital to insulin synthesis research. Thanks to his proposal, the synthetic insulin research continued smoothly and at last, the mission was accomplished in 1965.

Yinglai Wang organized several national training programs to educate young scientists in addition to establishing a strong biochemistry research team for CAS. Hundreds of young scientists benefited significantly from the systematic knowledge and classical experiments rendered through the combination of classroom instruction and benchmarking. In 1961, approximately 400 people attended the training programs. In 1979 and 1983, the number of participants increased to more than 500. Many outstanding Chinese biochemists were trained under these programs, including Academicians Zaiping Li, Genjun Xu, Fanhong Guo, and Xinyuan Liu, among others.

Aside from his endeavors as a leader and chief scientist, Yinglai Wang made significant contribution to the development of life sciences in China. He always gave top priority to the overall situation and national interests, and never considered personal gain or loss. Suffice to say, the achievements in biochemical research would not have been obtained if not for Yinglai Wang. However, he never affixed his name on the articles related to these achievements and even removed his name if it is cited in an article. In his opinion, "it is not that a penny saved is a penny earned" and only scientists whose name appears at the first line deserve to sign them. Such selflessness is worth the admiration!

Genjun Xu, a CAS academician, praised Yinglai Wang with a quotation from Tao Te Ching: "The top class of virtue is like water, which benefits ten thousand objects without any demands for return." This is an honest portrayal of the glorious life of Yinglai Wang.

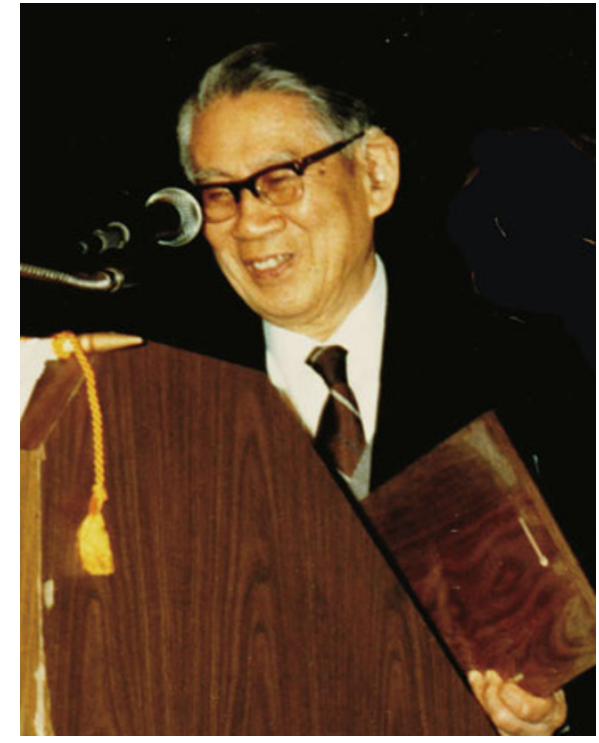

Dr. Yinglai Wang at the Miami Biotechnology Symposium in 1988

In 1988, Yinglai Wang received the "Life Achievement Award" at the Miami Biotechnology Symposium held in America. In 1996, a significant number of scientists nominated him for the Prize for Science and Technology Achievement of the Ho Leung Ho Lee Foundation. Nevertheless, he considered that, his achievements need not be mentioned. When he was told that he had won a million RMB for his contribution, he naively asked where this money would be used! He immediately sponsored a scholarship for his students in CAS. For Yinglai Wang, winning an award is an encouragement rather than a means for gaining individual accolade or money, and that his prize should no doubt be a reward not for him but for deserving students!

Seemingly, "Benefits ten thousand objects without any demands for return" was the basic philosophy that guided the life of Yinglai Wang. The maxim "dedication, realism, consolidation, courage" is not only the principle of instruction of the Shanghai Institute of Biochemistry at CAS, but a summation of a lifetime of painstaking efforts of Yinglai Wang. Regardless of the education of scientific talents or the accomplishments in scientific research project, his contributions to the strategic and perspective development will never lose their significance in the context of biochemical research in China. He deserves the respect for his greatness and goodness! 